Archive for

Organic Chemistry

Arkivoc 2017, part v, 67-79

\title{
Synthesis, anti-inflammatory activity of picen-13-ylmethylene derivatives
}

\author{
T. Shanmuganathan, ${ }^{* a, b}$ A. A. M. Prince, ${ }^{b}$ N. Dhatchanamoorthy, ${ }^{a}$ \\ K. Parthasarathy, ${ }^{c}$ and M. Venugopal ${ }^{d}$ \\ a Orchid Pharma Ltd, $R$ \& D Centre, Chennai 600119, India \\ ${ }^{b}$ Ramakrishna Mission Vivekananda College, Department of chemistry, Mylapore, \\ Chennai 600 004, India \\ c Siddha Central Research Institute, Central Council for Research in Siddha, \\ Chennai 600 106, India \\ ${ }^{d}$ Ven Biotech Private Limited, Chennai, India \\ Email: trshanmuganathan09@yahoo.com
}

Received 03-19-2017

Accepted 05-27-2017

Published on line 07-09-2017

\section{Abstract}

A series of picene-13-ylmethylene derivatives (11-17) were synthesized by Knoevenagel condensation of active methylene compounds with picene-13-carbaldehyde.
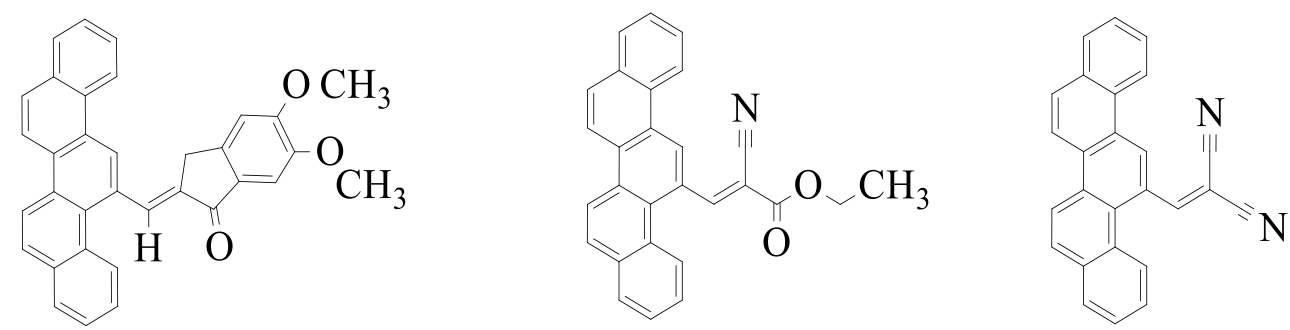

Keywords: Photo cyclization, picene-13-carbaldehyde, picene-13-ylmethylene derivatives, anti-inflammatory 


\section{Introduction}

Picene or benzo[a]chrysene, is well documented for use in material chemistry. ${ }^{1,2}$ Picene derivatives were found to be present in abundance as pentacyclic triterpenes and triterpenoids which are often bioactive and present a huge therapeutic potential. Many pentacyclic triterpenes namely oleane, oleanolic acid and ursane are reported to possess promising antitumor, ${ }^{3-5}$ antiviral, ${ }^{6}$ antidiabetic, ${ }^{7}$ anti-inflammatory ${ }^{8}$ activities. Also a derivative of picene, namely octadecahydro-picene-2,3,14,15-tetranone isolated and purified from the root bark of Zizyphus nummularia, reported to possess anti-cancer and anti-inflammatory activities. ${ }^{9,10}$

Many polycyclic aromatic compounds such as flavone, quercetin, chrysin and pyrimido[4,5-b]quinolines were reported to possess anti-inflammatory or antioxidant properties. ${ }^{11,12}$ Because of the close structural similarity with the reported polycyclic compounds, we have envisaged to explore the anti-inflammatory activity of picene analogues. Thus, our interest is to conjugate two naphthalene moieties by cyclization which results in the formation of a picene moiety. Substitution at its alpha position with various esters or amides leads to novel picene methylene derivatives in order to evaluate their biological properties. In order to accomplish our objective, we used a Knoevenagel condensation for the synthesis of novel structures incorporating both the picene moiety and several active methylene compounds, namely, ethyl cyanoacetate, malononitrile, cyanoacetamide, diethyl malonate, ethyl acetoacetate, acetylacetone and 5,6 dimethoxy-1indanone with picene-13-carbaldehyde. The synthesized picen-13-ylmethylene derivatives were characterized by NMR, IR, mass spectra, elemental analysis. The structures were confirmed by single crystal XRD of a selected example. The compounds were evaluated by in vitro biological tests for their anti-inflammatory properties.

\section{Results and Discussion}

In the present work, we synthesized a new series of picen-13-ylmethylene derivatives 11-17 by condensing active methylene groups with picene-13-carbaldehyde as shown in Scheme 1 and 2. The first step in the Scheme 1 was the condensation of 1-naphthaldehyde 1 with 1 -naphthyl acetic acid $\mathbf{2}$ in the presence of triethylamine and acetic anhydride produced 2,3-di(naphthalen-1-yl)acrylic acid 3 $^{13,14}$ with $62 \%$ yield after recrystallization from ethyl acetate.

Table 1. Reaction conditions and yield of $\mathbf{3}$ for the esterification using an alcohol

\begin{tabular}{lcccc}
\hline \multicolumn{1}{c}{ Solvent } & $\begin{array}{c}\text { Reaction } \\
\text { condition }\end{array}$ & $\begin{array}{c}\text { Reaction } \\
\text { Time }\end{array}$ & $\begin{array}{c}\text { Reaction } \\
\text { conversion }\end{array}$ & $\begin{array}{c}\text { Product Yield } \\
\%\end{array}$ \\
\hline Ethanol & Reflux & 3 days & $60 \%$ & $50 \%$ of compound $\mathbf{4}$ \\
$\begin{array}{l}\text { Mixture of Ethanol and Toluene } \\
\text { Methanol }\end{array}$ & Reflux & $10 \mathrm{hrs}$ & $100 \%$ & $86 \%$ of compound $\mathbf{4}$ \\
$\begin{array}{l}\text { Mixture of Methanol and } \\
\text { Toluene }\end{array}$ & Reflux & 3 days & $60 \%$ & $50 \%$ of compound $\mathbf{5}$ \\
\hline
\end{tabular}


Compound $\mathbf{3}$ was then converted to the corresponding ester $\mathbf{4} \& \mathbf{5}$ using ethanol or methanol and a catalytic amount of sulphuric acid. Esterification of compound $\mathbf{3}$ with ethanol and sulphuric acid under reflux condition over 3 days gave 50\% yield of compound 4 . As the starting compound $\mathbf{3}$ was insoluble in 25 volumes of ethanol under reflux condition, we conducted the experiment using toluene as solvent under Dean Stark conditions. Under continuous removal of water, the esterification reaction was completed in 10 hours with $91 \%$ yield of compound 4 without further purification. (Table 1)

The ester was cyclized to ethyl or methyl picene-13-carboxylate 6 \& $7^{15-19}$ under irradiation with UV light at $365 \mathrm{~nm}$ in the presence of iodine. We studied the oxidative cyclisation of compound 4 with several reactants under different reaction conditions and the results are summarized in Table 2. Treatment of compound $\mathbf{4}$ with aluminium chloride; or a mixture containing aluminium chloride and sodium chloride ${ }^{20}$ at $140{ }^{\circ} \mathrm{C}$; or aluminium chloride and stannic chloride ${ }^{21}$;or irradiation with $254 \mathrm{~nm}$ in the presence of iodine gave no product. Irradiation of compound 4 with $365 \mathrm{~nm}$ light without stirring condition gave 30\% yield. The previously reported process ${ }^{22}$ of vanadium trifluoride oxide in trifluoroacetic acid afforded picene-13-carboxylic acid methyl ester 7 with a yield of $47 \%$. In our present study, it was found that 1 mole of iodine and irradiation with light of $365 \mathrm{~nm}$ in benzene under stirring are necessary to produce compound 6 \& 7 in good yield (86\% \& $87 \%$ respectively) without chromatographic purification.

Table 2. Mole ratio of reactants, reaction conditions and yield for the oxidative cyclisation of Compound 4

\begin{tabular}{|c|c|c|c|c|}
\hline Reactant & Solvent & Temperature $\left({ }^{\circ} \mathrm{C}\right)$ & $\begin{array}{l}\text { Reaction time } \\
\text { (hrs.) }\end{array}$ & Yield (\%) of 6 \\
\hline $\begin{array}{l}\mathrm{AlCl}_{3} \text { (5 mol equiv) \& Sodium Chloride } \\
\text { (5 mol equiv) }\end{array}$ & Neat & $140^{\circ} \mathrm{C}$ & 6 & No product \\
\hline $\mathrm{AlCl}_{3}$ (5 mol equiv) & Benzene & Reflux & 6 & No product \\
\hline $\begin{array}{c}\mathrm{AlCl}_{3} \text { (5 mol equiv) \& Stannic Chloride } \\
\text { (2.5 mol equiv) }\end{array}$ & Benzene & Reflux & 6 & No product \\
\hline $\begin{array}{l}\text { lodine (1mol equiv); irradiation at } 254 \\
\mathrm{~nm}\end{array}$ & Benzene & $\begin{array}{l}25-30^{\circ} \mathrm{C} \text {, without } \\
\text { stirring }\end{array}$ & 12 & No product \\
\hline $\begin{array}{l}\text { lodine (1mol equiv); irradiation at } 254 \\
\mathrm{~nm}\end{array}$ & Benzene & $\begin{array}{l}25-30^{\circ} \mathrm{C} \text {, with } \\
\text { stirring }\end{array}$ & 12 & No product \\
\hline $\begin{array}{l}\text { lodine (1mol equiv); irradiation at } 365 \\
\mathrm{~nm}\end{array}$ & Benzene & $\begin{array}{l}25-30^{\circ} \mathrm{C} \text {, without } \\
\text { stirring }\end{array}$ & 12 & 30 \\
\hline $\begin{array}{l}\text { lodine (1mol equiv); irradiation at } 365 \\
\mathrm{~nm}\end{array}$ & Benzene & $\begin{array}{l}25-30{ }^{\circ} \mathrm{C} \text {, with } \\
\text { stirring }\end{array}$ & 12 & $86^{15-19}$ \\
\hline $\begin{array}{l}\text { lodine (3mol equiv); irradiation at } 500 \\
\mathrm{~nm}\end{array}$ & cyclohexane & Not reported & Not reported & $\begin{array}{c}31^{22,15} \\
\text { (after } \\
\text { column } \\
\text { chromatogra } \\
\text { phy) }\end{array}$ \\
\hline $\begin{array}{c}\text { Vanadium Trifluoride oxide (4.4 mol } \\
\text { equiv) }\end{array}$ & $\begin{array}{l}\text { Dichloromet } \\
\text { hane }\end{array}$ & $0{ }^{\circ} \mathrm{C}$ & Not reported & $47^{22}$ \\
\hline
\end{tabular}

The picene-13-carboxylic acid ethyl ester 6 was hydrolyzed with sodium hydroxide produced picene-13carboxylic acid 8 in $88 \%$ yield. 
Reduction of picene-13-carboxylic acid methyl ester with lithium aluminium hydride has been reported for the preparation of compound 9 with a yield of $97 \% .{ }^{23}$ In our study, mild reducing agents like di-isobutyl aluminium hydride [DIBAL (1M in Toluene)] was used to reduce picene-13-carboxylic acid ethyl ester 6 to picen-13-ylmethanol $\mathbf{9}^{24}$ in $90 \%$ yield. It was found that the oxidation of compound 9 with manganese dioxide $^{11}$ in dichloromethane was not completed even under reflux condition. Therefore, we tried pyridinium chlorochromate as an oxidising agent in dichloromethane at $25-30{ }^{\circ} \mathrm{C}$ and obtained the compound $10^{25,26}$ in $87 \%$ yield without further purification. The picene-13-carbaldehyde 10 was condensed with active methylene compounds using piperidine as a base to give the title compounds $11-17.27,28$

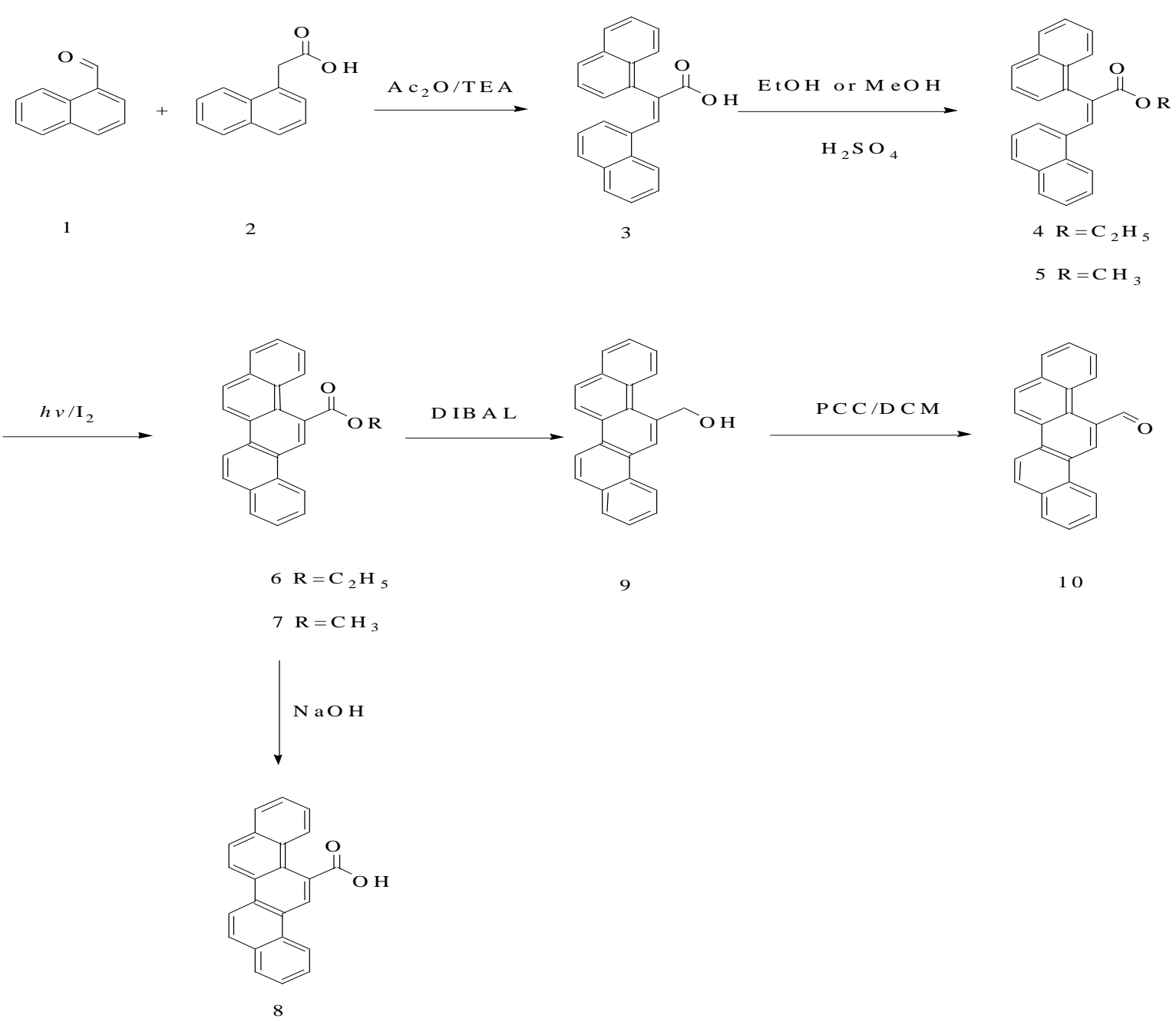

Scheme 1. Synthetic route for Picene-13-carboxylic acid (8) and Picene-13-carbaldehyde (10). 


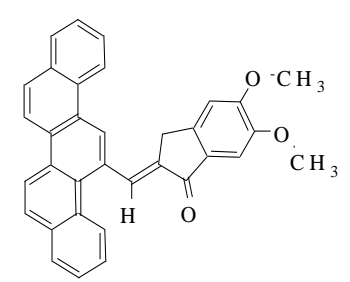

17

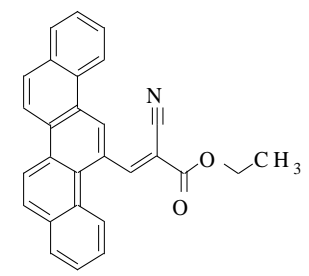

11

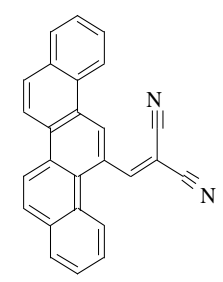

12
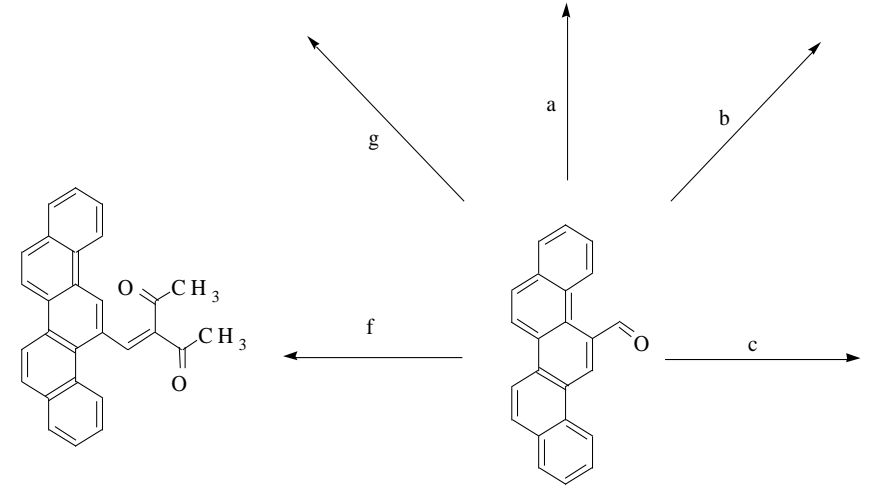

10

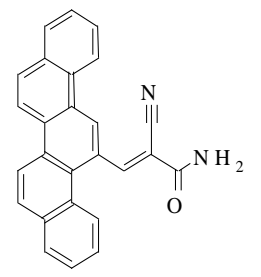

13
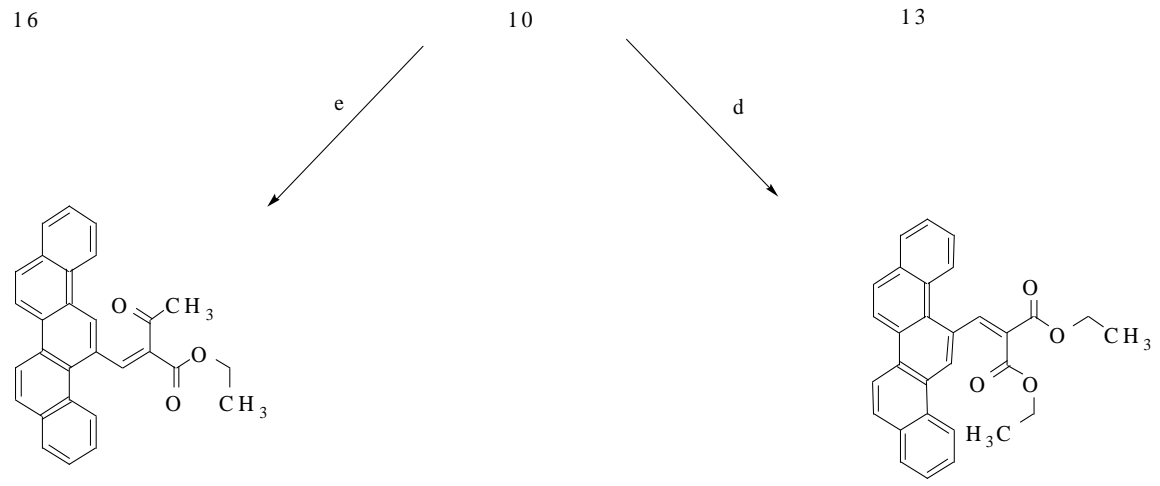

14

Scheme 2. Synthetic route for various picen-13-ylmethylene derivatives (11-17) a) ethyl cyanoacetate b) malononitrile c) cyanoacetamide d) diethylmalonate e) ethyl acetoacetate f) acetyl acetone and g) 5,6 dimethoxy-1-indanone.

All the synthesized compounds were characterized by ${ }^{1} \mathrm{H} N M R,{ }^{13} \mathrm{C} N M R, \mathrm{IR}$ spectroscopy, Mass and elemental analysis. The structure of $\mathbf{1 1}$ was confirmed by single crystal X-ray diffractogram (CCDC No. CCDC 1400968). Based on the single crystal structure, the configuration of the compound was confirmed as the $E$ isomer. The crystal parameters for compound $\mathbf{1 1}$ are given in Table $\mathbf{3}$ and the ORTEP diagram is shown in Figure 1. 


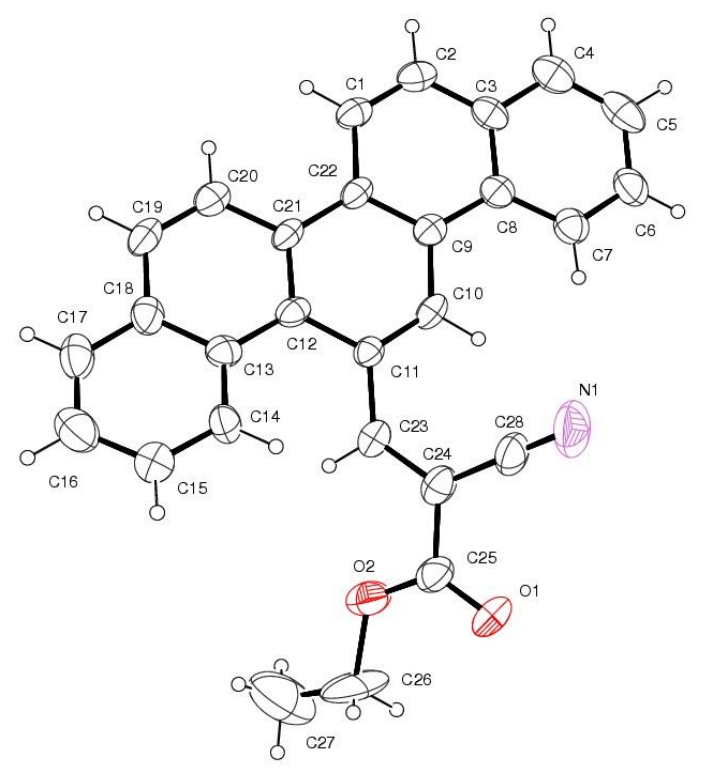

Figure 1. ORTEP diagram of Compound 11.

Table 3. Crystal data and structure refinement for Compound 11

\begin{tabular}{ll}
\hline Empirical formula & $\mathrm{C}_{28} \mathrm{H}_{19} \mathrm{NO}_{2}$ \\
Formula weight & 402.1 \\
Temperature & $296(2) \mathrm{K}$ \\
Wavelength & $0.71073 \AA$ \\
Crystal system, space group & Triclinic, $\mathrm{P}-1$ \\
& $\mathrm{a}=11.4481(11) \AA$ alpha $=108.889(3)^{\circ}$ \\
Unit cell dimensions & $\mathrm{b}=12.8106(11) \AA$ beta $=97.646(3)^{\circ}$ \\
& $\mathrm{c}=15.4300(13) \AA$ gamma $=90.531(3)^{\circ}$ \\
Volume & $2118.7(3) \AA^{3}$ \\
Z, Calculated density & $2,1.259 \mathrm{~g} / \mathrm{cm}^{3}$ \\
Absorption coefficient & $0.079 \mathrm{~mm} \mathrm{~m}^{-1}$ \\
F(000) & 840 \\
Crystal size & $0.210 \times 0.150 \times 0.100 \mathrm{~mm}$ \\
Theta range for data collection & 1.409 to $21.57^{\circ}$ \\
Limiting indices & $-11<=\mathrm{h}<=11,-13<=\mathrm{k}<=13,-15<=\mathrm{l}<=15$ \\
Reflections collected / unique & $18796 / 4896[\mathrm{R}(\mathrm{int})=0.0741]$ \\
Completeness to theta $=$ & 21.570 \\
Absorption correction & $\mathrm{None}$ \\
Refinement method & $\mathrm{Full}-\mathrm{matrix}$ least-squares on $\mathrm{F}^{2}$ \\
Data / restraints / parameters & $4896 / 0 / 561$ \\
Goodness-of-fit on F^2 & 1.007 \\
Final R indices [l>2sigma(I)] & $\mathrm{R} 1=0.0735, \mathrm{wR} 2=0.2161$ \\
R indices (all data) & $\mathrm{R} 1=0.1930, \mathrm{wR} 2=0.3213$ \\
Extinction coefficient & $\mathrm{n} / \mathrm{a}$ \\
Largest diff. peak and hole & 0.391 and $-0.181 \mathrm{e} \AA^{-3}$ \\
\hline
\end{tabular}


Anti-inflammatory activity. The synthesized compounds were screened for in vitro anti-inflammatory activity using the inhibition of bovine serum albumin denaturation method. From the activity data, the antiinflammatory activity screening revealed that compounds containing the active methylene groups such as diethylmalonate 14, acetylacetone 16, and 5,6 dimethoxy-1-indanone 17 attached to a picene-13carbaldehyde moiety exhibited the biggest potent anti-inflammatory activity comparable to diclofenac sodium as a reference standard. In addition the data indicated that, the cyano ester 11 or cynamide 13, malononitrile 12 and ethyl acetoacetate ester 15 of picene-13-ylmethylene are comparatively less potent than its malonate $\mathbf{1 4}$ or dione $\mathbf{1 6}$ or indanone $\mathbf{1 7}$ derivatives and moderate activity in comparison to diclofenac sodium (Table 4).

Table 4. In vitro anti-inflammatory activity of compounds 6-10 \& 11-17 by inhibition of protein (Bovine serum albumin) denaturation method

\begin{tabular}{ccccc}
\hline \multirow{2}{*}{ Compound } & \multicolumn{3}{c}{ Activity (\% inhibition of protein denaturation) } \\
\cline { 2 - 5 } & $50 \mu \mathrm{g} / \mathrm{mL}$ & $100 \mu \mathrm{g} / \mathrm{mL}$ & $400 \mu \mathrm{g} / \mathrm{mL}$ & $800 \mu \mathrm{g} / \mathrm{mL}$ \\
\hline $\mathbf{7}$ & 15.81 & 27.02 & 35.91 & 50.88 \\
$\mathbf{8}$ & 14.82 & 25.26 & 32.55 & 48.88 \\
$\mathbf{9}$ & 10.59 & 26.72 & 45.64 & 62.41 \\
10 & 14.02 & 22.51 & 35.86 & 48.86 \\
11 & 14.28 & 24.85 & 47.21 & 52.34 \\
12 & 17.97 & 29.81 & 38.82 & 55.69 \\
13 & 16.61 & 29.42 & 37.88 & 54.65 \\
14 & 13.21 & 27.11 & 32.95 & 53.21 \\
15 & 30.32 & 50.15 & 70.32 & 84.48 \\
16 & 11.09 & 21.89 & 32.24 & 49.13 \\
17 & 27.81 & 49.62 & 64.99 & 80.38 \\
Std & 27.88 & 38.93 & 66.12 & 78.65 \\
\hline
\end{tabular}

Std - Diclofenac sodium.

\section{Conclusions}

A new versatile method with improved yield for the photocyclisation of 2,3-di(naphthalen-1-yl)acrylic acid ester was reported using mild conditions i.e., UV irradiation. A new series of picene-13-ylmethylene derivatives were synthesized, characterized and evaluated for in vitro anti-inflammatory activity. Among the picene-13-ylmethylene derivatives, compounds 14, 16 and 17 possess promising anti-inflammatory activity compared to the reference drug diclofenac sodium. 


\section{Experimental Section}

General. All the chemicals and reagents used were lab grade material procured from Alfa aesar. The melting points were determined using Buchi apparatus by the open capillary tube method. The IR spectra were recorded in Perkin-Elmer series 2000 FTIR spectrophotometer using $\mathrm{KBr}$ pellet. ${ }^{1} \mathrm{H}$ NMR and ${ }^{13} \mathrm{C}$ NMR spectra were obtained in $\mathrm{CDCl}_{3}, \mathrm{DMSO}-d_{6}$ on a Bruker spectrometer at 400 and $100 \mathrm{MHz}$, respectively. The chemical shifts are reported in ppm $(\delta)$ relative to tetramethylsilane as internal standard, coupling constants $(J)$ are in hertz $(\mathrm{Hz})$. Mass were recorded on ESI - Perkin Elmer Sciex, API 3000 mass spectrometer. Pre-coated silica gel $\mathrm{GF}_{254}$ plates from Merck were used for thin layer chromatography. The elemental analyses were recorded in Thermo Finnigan Flash EA 1112 elemental analyser.

2,3-Di(naphthalen-1-yl)acrylic acid (3). A mixture of 1-naphthaldehyde 1 (0.1 mole), 1-naphthylacetic acid 2 (0.1 mole), triethylamine $(10 \mathrm{~mL})$ and acetic anhydride $(20 \mathrm{~mL})$ was heated at reflux over 15 hours. The reaction progress was monitored using TLC (hexane: ethyl acetate; 7:3). After completion, the reaction mixture was poured into $200 \mathrm{~mL}$ water and extracted with ethyl acetate $(2 \times 250 \mathrm{~mL})$. The combined organic layer was washed with water and evaporated. The residue was triturated with $200 \mathrm{~mL}$ of ethyl acetate: heptane mixture in the ratio of 10: 90 produced $28 \mathrm{~g}$ of the 2,3-di(naphthalen-1-yl)acrylic acid which was recrystallized from ethyl acetate afforded the title compound $(20 \mathrm{~g}, 62 \%)$ as a Pale yellow powder. $\mathrm{mp} 232-$ $235^{\circ} \mathrm{C}$ (lit. ${ }^{13}: 227-228{ }^{\circ} \mathrm{C} \&$ lit. $^{14}{ }^{14}: 232-233^{\circ} \mathrm{C}$ ). IR (solid, $\mathrm{KBr}, \mathrm{vmax}, \mathrm{cm}^{-1}$ ): 3435, 3054, 1674, 1506, 1423, 1275, 800, 777. ${ }^{1} \mathrm{H}$ NMR $\left(400 \mathrm{MHz}, \mathrm{CDCl}_{3}\right): \delta_{\mathrm{H}} 8.85(\mathrm{~s}, 1 \mathrm{H}), 8.26(\mathrm{~d}, J 8.4 \mathrm{~Hz}, 1 \mathrm{H}), 7.91(\mathrm{~d}, J 7.8 \mathrm{~Hz}, 1 \mathrm{H}), 7.83(\mathrm{~d}, J 7.6$ $\mathrm{Hz}, 1 \mathrm{H}), 7.78(\mathrm{t}, J 8.6 \mathrm{~Hz}, 2 \mathrm{H}), 7.60(\mathrm{t}, J 8.5 \mathrm{~Hz}, 2 \mathrm{H}), 7.51(\mathrm{t}, J 7.4 \mathrm{~Hz}, 1 \mathrm{H}), 7.46-7.39(\mathrm{~m}, 2 \mathrm{H}), 7.31-7.27(\mathrm{~m}, 1 \mathrm{H})$, $7.20(\mathrm{~d}, J 6.9 \mathrm{~Hz}, 1 \mathrm{H}), 6.93(\mathrm{t}, J 7.6 \mathrm{~Hz}, 1 \mathrm{H}), 6.81(\mathrm{~d}, J 7.2 \mathrm{~Hz}, 1 \mathrm{H}) .{ }^{13} \mathrm{C} \mathrm{NMR}\left(100 \mathrm{MHz}, \mathrm{CDCl}_{3}+\mathrm{DMSO} d_{6}\right): \delta_{c}$ $170.1,140.2$, 133.9, 133.8, 133.6, 133.3, 132.5, 132.0, 131.9, 129.1, 128.7, 128.5, 128.2, 127.8, 127.2, 126.6, 126.4, 126.0, 125.9, 125.6, 125.4, 125.1, 124.0. ESI-MS m/z calcd 324.1. Found: $325.0[\mathrm{M}+\mathrm{H}]^{+}, 342.0[\mathrm{M}+\mathrm{NH}]^{+}$. Anal. calcd for $\mathrm{C}_{23} \mathrm{H}_{16} \mathrm{O}_{2}$ : C, 85.16; $\mathrm{H}, 4.97$. Found: $\mathrm{C}, 85.12 ; \mathrm{H}, 5.06$.

Ethyl 2,3-di(naphthalen-1-yl)acrylate (4). 2,3-Di(naphthalen-1-yl)acrylic acid 3 (0.03 mole), ethanol (75 mL) and sulphuric acid ( 0.03 mole) was heated at reflux in toluene $(50 \mathrm{~mL})$ under a Dean-Stark water separator over 10 hours. The reaction was monitored using TLC (hexane: ethyl acetate; 7:3). After completion, the reaction mixture was evaporated under vacuum and the product was extracted with ethyl acetate $(200 \mathrm{~mL})$. The ethyl acetate layer was washed with water, aqueous sodium hydroxide solution, water and evaporated under vacuum. The residue was triturated with hexane $(50 \mathrm{~mL})$, filtered and dried at $50{ }^{\circ} \mathrm{C}$ under vacuum afforded the title compound $(9.9 \mathrm{~g}, 91 \%)$ as a Off-white powder. $\mathrm{mp} 115-117{ }^{\circ} \mathrm{C}$. IR (solid, $\mathrm{KBr}, \mathrm{vmax}, \mathrm{cm}^{-1}$ ): $3433,3055,2974,1705,1626,1504,1271,801,774 .{ }^{1} \mathrm{H} N M R\left(400 \mathrm{MHz}, \mathrm{CDCl}_{3}\right): \delta_{\mathrm{H}} 8.79(\mathrm{~s}, 1 \mathrm{H}), 8.25(\mathrm{~d}, J 8.4$ $\mathrm{Hz}, 1 \mathrm{H}), 7.88-7.83(\mathrm{~m}, 2 \mathrm{H}), 7.79(\mathrm{t}, J 8.9 \mathrm{~Hz}, 2 \mathrm{H}), 7.61-7.39(\mathrm{~m}, 5 \mathrm{H}), 7.29(\mathrm{t}, J 7.1 \mathrm{~Hz}, 1 \mathrm{H}), 7.16-7.14(\mathrm{~m}, 1 \mathrm{H}$,$) ,$ $6.94(\mathrm{t}, J 7.6 \mathrm{~Hz}, 1 \mathrm{H}), 6.79(\mathrm{~d}, J 7.3 \mathrm{~Hz}, 1 \mathrm{H}), 4.32$ (quartet, J $7.1 \mathrm{~Hz}, 2 \mathrm{H}), 1.22$ (t, J $7.1 \mathrm{~Hz}, 3 \mathrm{H}) .{ }^{13} \mathrm{C} \mathrm{NMR}(100 \mathrm{MHz}$, $\left.\mathrm{CDCl}_{3}\right): \delta_{c} 168.1,140.0,134.0,133.9,133.6,133.4,132.7,132.1,131.9,129.2,128.8,128.6,128.3,127.9$, 127.4, 126.7, 126.4, 126.1, 125.9, 125.7, 125.4, 125.2, 124.1, 61.4, 14.4. ESI-MS m/z calcd 352.1. Found: 353.1 $[\mathrm{M}+\mathrm{H}]^{+}$. Anal. calcd for $\mathrm{C}_{25} \mathrm{H}_{20} \mathrm{O}_{2}: \mathrm{C}, 85.20, \mathrm{H}, 5.72$. Found: $\mathrm{C}, 85.16 ; \mathrm{H}$ 5.76.

Methyl 2,3-di(naphthalen-1-yl)acrylate (5). 2,3-Di(naphthalen-1-yl)acrylic acid (3) (0.012 mole), methanol (60 $\mathrm{mL}$ ) and sulphuric acid (0.012 mole) was heated at reflux in toluene (40 mL) under a Dean-Stark water separator over 10 hours. The reaction was monitored using TLC (hexane: ethyl acetate; 7:3). After completion, the reaction mixture was evaporated under vacuum and the product was extracted with ethyl acetate (100 $\mathrm{mL}$ ). The ethyl acetate layer was washed with water, aqueous sodium hydroxide, water and evaporated under vacuum. The residue was triturated with hexane $(20 \mathrm{~mL})$, filtered and dried at $50{ }^{\circ} \mathrm{C}$ under vacuum afforded 
the title compound $(4.0 \mathrm{~g}, 96 \%)$ as a Pale yellow powder. $\mathrm{mp} 135-137^{\circ} \mathrm{C}$. IR (solid, $\mathrm{KBr}, \mathrm{vmax}, \mathrm{cm}^{-1}$ ): 3401 , 2045, 2941, 1709, 1628, 1505, 1425, 1275, 801, 763. ${ }^{1} \mathrm{H}$ NMR $\left(400 \mathrm{MHz}, \mathrm{CDCl}_{3}\right): \delta_{\mathrm{H}} 8.82(\mathrm{~s}, 1 \mathrm{H}), 8.25(\mathrm{~d}, J 8.4$ $\mathrm{Hz}, 1 \mathrm{H}), 7.86(\mathrm{t}, J 6.8 \mathrm{~Hz}, 2 \mathrm{H}), 7.80(\mathrm{t}, J 7.0 \mathrm{~Hz}, 2 \mathrm{H}), 7.62-7.58,(\mathrm{~m}, 2 \mathrm{H}), 7.53-7.41(\mathrm{~m}, 3 \mathrm{H}), 7.31(\mathrm{t}, J 7.2 \mathrm{~Hz}, 1 \mathrm{H})$, $7.18(\mathrm{~d}, J 7.0 \mathrm{~Hz}, 1 \mathrm{H}), 6.94(\mathrm{t}, J 7.6 \mathrm{~Hz}, 1 \mathrm{H}), 6.79(\mathrm{~d}, J 7.2 \mathrm{~Hz}, 1 \mathrm{H}), 3.77(\mathrm{~s}, 3 \mathrm{H}) .{ }^{13} \mathrm{C} \mathrm{NMR}\left(100 \mathrm{MHz}^{\mathrm{C} C D C l}\right): \delta_{c}$ $168.7,140.4,133.8,133.7,133.5,133.4,132.7,132.2,131.8,129.3,128.8,128.7,128.4,127.9,127.4,126.8$, 126.6, 126.2, 126.0, 125.7, 125.3, 125.2, 124.1, 52.7. ESI-MS $\mathrm{m} / \mathrm{z}$ calcd 338.1. Found: $339.2[\mathrm{M}+\mathrm{H}]^{+}, 356.2$ $\left[\mathrm{M}+\mathrm{NH}_{4}\right]^{+}$. Anal. calcd for $\mathrm{C}_{24} \mathrm{H}_{18} \mathrm{O}_{2}: \mathrm{C}, 85.18 ; \mathrm{H}, 5.36$. Found: $\mathrm{C}, 85.16 ; \mathrm{H}, 5.26$.

Ethyl picene-13-carboxylate (6). A solution of compound 4 (0.006 mole) and iodine (0.006 mole) in benzene $(25 \mathrm{~mL})$ was stirred under irradiation with $365 \mathrm{~nm}$ light in a water cooled quartz immersion well for 12 hours. The completion of reaction was monitored using TLC (hexane: ethyl acetate 7:3). After completion, the reaction mass was washed with sodium sulphite solution, water and dried with anhydrous sodium sulfate. The solvent was evaporated and triturated with cyclohexane $(5 \mathrm{~mL})$, filtered and dried at $50{ }^{\circ} \mathrm{C}$ under vacuum afforded the title compound $\left(1.8 \mathrm{~g}, 86 \%\right.$ ) as a Off-white powder. $\mathrm{mp} 172-174{ }^{\circ} \mathrm{C}$. IR (solid, $\mathrm{KBr}, \mathrm{vmax}, \mathrm{cm}^{-1}$ ): 3427, 2975, 1713, 1604, 1445, 1265, 808, 775. ${ }^{1} \mathrm{H}$ NMR $\left(400 \mathrm{MHz}, \mathrm{CDCl}_{3}\right): \delta_{\mathrm{H}} 9.07(\mathrm{~s}, 1 \mathrm{H}), 8.83(\mathrm{~d}, J 8.3 \mathrm{~Hz}, 1 \mathrm{H})$, $8.74(\mathrm{~d}, J 9.2 \mathrm{~Hz}, 1 \mathrm{H}), 8.71(\mathrm{~d}, J 9.3 \mathrm{~Hz}, 1 \mathrm{H}), 8.33(\mathrm{~d}, J 8.0 \mathrm{~Hz}, 1 \mathrm{H}), 8.06(\mathrm{t}, J 8.8 \mathrm{~Hz}, 2 \mathrm{H}), 7.99(\mathrm{~d}, J 8.1 \mathrm{~Hz}, 2 \mathrm{H})$, $7.76(\mathrm{t}, J 8.1 \mathrm{~Hz}, 1 \mathrm{H}), 7.68-7.58(\mathrm{~m}, 3 \mathrm{H}), 4.57$ (quartet, J $7.1 \mathrm{~Hz}, 2 \mathrm{H}), 1.33(\mathrm{t}, J 7.1 \mathrm{~Hz}, 3 \mathrm{H}) ;{ }^{13} \mathrm{C} \mathrm{NMR}(100 \mathrm{MHz}$, $\left.\mathrm{CDCl}_{3}\right): \delta_{c} 172.6,132.9,132.2,130.6,130.0,129.9,129.5,129.4,128.8,128.7,128.5,127.6,127.5,127.2$, $126.9,126.1,125.9,124.2,123.4,121.5,121.4,62.2,14.2 ;$ ESI-MS $m / z$ calcd 350.1. Found: $351.2[\mathrm{M}+\mathrm{H}]^{+}, 368.1$ $\left[\mathrm{M}+\mathrm{NH}_{4}\right]^{+}$. Anal. calcd for $\mathrm{C}_{25} \mathrm{H}_{18} \mathrm{O}_{2}: \mathrm{C}, 85.69 ; \mathrm{H}, 5.18$. Found: $\mathrm{C}, 85.72 ; \mathrm{H} 5.10$.

Methyl picene-13-carboxylate (7). A solution of compound 5 ( 0.003 mole) and iodine ( 0.003 mole) in benzene $(25 \mathrm{~mL})$ was stirred using magnetic stirrer under irradiation with $365 \mathrm{~nm}$ light in a water cooled quartz immersion well for 12 hours. The completion of reaction was monitored using TLC (hexane: ethyl acetate 7:3). The reaction mass was washed with sodium sulphite solution, water and dried with anhydrous sodium sulfate. The solvent was evaporated and triturated with cyclohexane $(5 \mathrm{~mL})$, afforded the title compound $(0.85 \mathrm{~g}, 87 \%)$ as a Colorless powder. $\mathrm{mp} 227-230{ }^{\circ} \mathrm{C}$. [lit. ${ }^{22}$ : 230-233 ${ }^{\circ} \mathrm{C}$ ]. IR (solid, $\mathrm{KBr}$, vmax, $\mathrm{cm}^{-1}$ ): $3416,2945,1799,1604$, 1435, 1264, 808, 765. ${ }^{1} \mathrm{H}$ NMR $\left(400 \mathrm{MHz}, \mathrm{CDCl}_{3}\right): \delta_{\mathrm{H}} 9.09(\mathrm{~s}, 1 \mathrm{H}), 8.84(\mathrm{~d}, J 8.3 \mathrm{~Hz}, 1 \mathrm{H}), 8.77-8.72(\mathrm{~m}, 2 \mathrm{H}), 8.27$ (d, J $7.3 \mathrm{~Hz}, 1 \mathrm{H}), 8.10(\mathrm{t}, J 9.9 \mathrm{~Hz}, 2 \mathrm{H}), 8.02(\mathrm{~d}, J 8.0 \mathrm{~Hz}, 2 \mathrm{H}), 7.78(\mathrm{t}, J 7.2 \mathrm{~Hz}, 1 \mathrm{H}), 7.68-7.62(\mathrm{~m}, 3 \mathrm{H}), 4.03(\mathrm{~s}$, 3H). ${ }^{13} \mathrm{C} \mathrm{NMR}\left(100 \mathrm{MHz}, \mathrm{CDCl}_{3}\right): \delta_{\mathrm{c}} 173.0,132.9,132.2,130.6,130.0,129.5,129.4,129.0,128.8,128.6,127.5$, 127.4, 127.3, 126.9, 126.8, 126.1, 124.3, 123.4, 121.5, 121.4, 52.9. ESI-MS m/z calcd 336.1. Found: 337.2 $[\mathrm{M}+\mathrm{H}]^{+}, 354.2\left[\mathrm{M}+\mathrm{NH}_{4}\right]^{+}$. Anal. calcd for $\mathrm{C}_{24} \mathrm{H}_{16} \mathrm{O}_{2}: \mathrm{C}, 85.69 ; \mathrm{H}, 4.79$. Found: $\mathrm{C}, 85.56 ; \mathrm{H}, 4.76$.

Picene-13-carboxylic acid (8). A mixture of compound 6 (0.001 mole), tetrahydrofuran $(8 \mathrm{~mL}), \mathrm{water}(2 \mathrm{~mL})$ and sodium hydroxide ( 0.001 mole) was heated to reflux over 8 hours. The completion of the reaction was checked using TLC (hexane: ethyl acetate 1:1). The mass was acidified with concentrated hydrochloric acid and the product was extracted with ethyl acetate $(50 \mathrm{~mL})$. The ethyl acetate layer was washed with water and evaporated. The residual mass was triturated with cyclohexane $(5 \mathrm{~mL})$, filtered and dried at $50{ }^{\circ} \mathrm{C}$ under vacuum afforded the title compound $(0.28 \mathrm{~g}, 88 \%)$ as a Pale brown powder. $\mathrm{mp} 267-270{ }^{\circ} \mathrm{C}$. IR (solid, $\mathrm{KBr}$, vmax, $\mathrm{cm}^{-1}$ ): 3435, 3206, 1676, 1425, 1252, 806,776. ${ }^{1} \mathrm{H}$ NMR $\left(400 \mathrm{MHz}, \mathrm{DMSO}-d_{6}\right): \delta_{\mathrm{H}} 13.71(\mathrm{~s}, 1 \mathrm{H}), 9.13(\mathrm{~s}$, $1 \mathrm{H})$, 9.04-8.97 (m, 3H), 8.62-8.59 (m, $1 \mathrm{H}), 8.24-8.21(\mathrm{~m}, 2 \mathrm{H}), 8.18-8.15(\mathrm{~m}, 2 \mathrm{H}), 7.84-7.76(\mathrm{~m}, 2 \mathrm{H}), 7.74-7.70$ $(\mathrm{m}, 2 \mathrm{H}) .{ }^{13} \mathrm{C}$ NMR $\left(100 \mathrm{MHz}, \mathrm{DMSO}-d_{6}\right): \delta_{c} 172.9,132.3,131.7,131.1,129.7,129.4,129.2,128.8,128.7,128.5$, $127.8,127.7,127.5,127.1,126.9,126.3,126.2,124.7,123.6,122.5,122.4,121.9,121.7$. ESI-MS $\mathrm{m} / \mathrm{z} \mathrm{calcd}$ 322.1. Found: $340.2\left[\mathrm{M}+\mathrm{NH}_{4}\right]^{+}, 321.2[\mathrm{M}-\mathrm{H}]^{-}$. Anal. calcd for $\mathrm{C}_{23} \mathrm{H}_{14} \mathrm{O}_{2}: \mathrm{C}, 85.70 ; \mathrm{H}, 4.38$. Found: $\mathrm{C}, 85.74 ; \mathrm{H}$, 4.36 .

Picen-13-ylmethanol (9). A solution of di-isobutyl aluminium hydride (1M in toluene $20 \%, 30 \mathrm{~mL}$ ) was added slowly to a solution of compound 6 (0.007 mole) in dichloromethane $(50 \mathrm{~mL})$ at $-75{ }^{\circ} \mathrm{C}$ under nitrogen 
atmosphere over 30 minutes. The progress of the reaction was monitored by TLC. (hexane: ethyl acetate 7:3). After completion, the mass was quenched with methanol $(10 \mathrm{~mL})$ followed by the addition of $1: 1$ aqueous hydrochloric acid $(20 \mathrm{~mL})$ at $-70{ }^{\circ} \mathrm{C}$. The product was extracted with ethyl acetate $(100 \mathrm{~mL})$ and washed with water. The solvent was evaporated and triturated with toluene $(40 \mathrm{~mL})$, filtered and dried at $50{ }^{\circ} \mathrm{C}$ under vacuum afforded the title compound $(1.94 \mathrm{~g}, 90 \%)$ as a Colorless powder. $\mathrm{mp} 192-195{ }^{\circ} \mathrm{C}$. [lit. ${ }^{23} 189.4{ }^{\circ} \mathrm{C}$ ]. IR (solid, $\mathrm{KBr}$, vmax, cm${ }^{-1}$ ): 3689, 3307, 3049, 2912, 1606, 1445, 1261, 1020, 977, 887, $803 \mathrm{~cm}^{-1} .{ }^{1} \mathrm{H}$ NMR (400 $\left.\mathrm{MHz}, \mathrm{DMSO}-d_{6}\right): \delta_{\mathrm{H}} 9.23(\mathrm{~s}, 1 \mathrm{H}), 9.09(\mathrm{~d}, J 8.1 \mathrm{~Hz}, 1 \mathrm{H}), 9.00-8.94(\mathrm{~m}, 3 \mathrm{H}), 8.15-8.12(\mathrm{~m}, 4 \mathrm{H}), 7.84-7.74(\mathrm{~m}, 4 \mathrm{H})$, $5.96(\mathrm{t}, J 5.2 \mathrm{~Hz}, 1 \mathrm{H}), 5.38(\mathrm{~d}, J 5.2 \mathrm{~Hz}, 2 \mathrm{H}) .{ }^{13} \mathrm{C}$ NMR $\left(100 \mathrm{MHz}, \mathrm{DMSO}-d_{6}\right): \delta_{c} 137.3,132.6,131.6,129.9,129.6$, $129.5,128.6,128.4,128.3,128.2,127.7,127.5,127.4,127.3,127.1,126.9,126.4,126.2,124.2,123.2,122.2$, 121.9, 65.0. ESI-MS $\mathrm{m} / \mathrm{z}$ calcd 308.1. Found: 367.1 [M+CH $\mathrm{CHOO}_{3}{ }^{-}$. Anal. calcd for $\mathrm{C}_{23} \mathrm{H}_{16} \mathrm{O}: \mathrm{C}, 89.58 ; \mathrm{H}, 5.23$. Found: C, 89.46; $H, 5.36$.

Picene-13-carbaldehyde (10). A suspension of compound 9 (1 g, 0.003 mole) in dichloromethane (60 mL) was added portionwise to a stirred mixture of pyridinium chlorochromate $(1.04 \mathrm{~g}, 0.005 \mathrm{~mole})$ in dichloromethane $(20 \mathrm{~mL})$ at $25-30{ }^{\circ} \mathrm{C}$. The reaction was monitored using TLC (hexane: ethyl acetate 7:3). The mass was filtered through celite and the filtrate was washed with water, aqueous sodium bicarbonate followed by water. The dichloromethane was evaporated and triturated with ethyl acetate $(20 \mathrm{~mL})$, filtered and dried at $50{ }^{\circ} \mathrm{C}$ under vacuum afforded the title compound $(0.86 \mathrm{~g}, 87 \%)$ as a Pale brown powder. $\mathrm{mp} 181-184{ }^{\circ} \mathrm{C}$. IR (solid, $\mathrm{KBr}$, vmax, $\left.\mathrm{cm}^{-1}\right)$ : 3435, 3053, 2923, 1678, 1604, 1377, 1262, 799, $773 \mathrm{~cm}^{-1} .{ }^{1} \mathrm{H}$ NMR $\left(400 \mathrm{MHz}, \mathrm{CDCl}_{3}\right): \delta_{\mathrm{H}} 10.75(\mathrm{~s}$, $1 \mathrm{H}), 9.39(\mathrm{~s}, 1 \mathrm{H}), 8.94(\mathrm{~d}, J 8.2 \mathrm{~Hz}, 1 \mathrm{H}), 8.80-8.73(\mathrm{~m}, 2 \mathrm{H}), 8.75(\mathrm{~d}, J 9.1 \mathrm{~Hz}, 1 \mathrm{H}), 8.18-8.10(\mathrm{~m}, 4 \mathrm{H}), 8.03(\mathrm{~d}, J 7.8$ $\mathrm{Hz}, 1 \mathrm{H}), 7.92-7.69(\mathrm{~m}, 4 \mathrm{H}) .{ }^{13} \mathrm{C} \mathrm{NMR}\left(100 \mathrm{MHz}, \mathrm{CDCl}_{3}\right): \delta_{\mathrm{c}} 193.3,133.1,133.0,132.0,131.1,130.4,130.0$, $129.3,128.7,128.6,128.5,127.9,127.7,127.6,127.4,127.3,126.7,124.6,123.6,121.6,121.2$. ESI-MS m/z calcd 306.1. Found: $307.2[\mathrm{M}+\mathrm{H}]^{+}$. Anal. calcd for $\mathrm{C}_{23} \mathrm{H}_{14} \mathrm{O}: \mathrm{C}, 90.17 ; \mathrm{H}, 4.61$. Found: $\mathrm{C}, 90.16 ; \mathrm{H}, 4.58$.

\section{General procedure for the synthesis of Picen-13-ylmethylene derivatives (11-17).}

A mixture of compound 10 (0.001 mole), active methylene compound scheme 2 (a-h) (0.001 mole) in ethanol $(5 \mathrm{~mL})$ was heated under reflux for 3 hours using piperidine $(0.5 \mathrm{~mL})$ as a catalyst. The progress of the reaction was monitored using TLC (hexane: ethyl acetate 7:3). The reaction mass was cooled to $25-30{ }^{\circ} \mathrm{C}$ and the product was collected by filtration and recrystallized from ethanol afforded compounds 11-17.

(E)-Ethyl 2-cyano-3-(picen-13-yl) acrylate (11). Prepared from 10 and ethyl cyanoacetate. Yellow powder, Yield: $80 \%$. $\mathrm{mp} 199-20{ }^{\circ} \mathrm{C}$. IR (solid, $\mathrm{KBr}, \mathrm{vmax}, \mathrm{cm}^{-1}$ ): 3431, 3053, 2975, 2228, 1718, 1593, 1264, 1095, 803 $\mathrm{cm}^{-1} .{ }^{1} \mathrm{H}$ NMR $\left(400 \mathrm{MHz}, \mathrm{CDCl}_{3}\right): \delta_{\mathrm{H}} 9.53(\mathrm{~s}, 1 \mathrm{H}), 9.12(\mathrm{~s}, 1 \mathrm{H}), 9.08(\mathrm{~d}, J 9.3 \mathrm{~Hz}, 1 \mathrm{H}), 9.03(\mathrm{t}, J 9.0 \mathrm{~Hz}, 2 \mathrm{H}), 8.41(\mathrm{~d}$, J $9.0 \mathrm{~Hz}, 1 \mathrm{H}), 8.31-8.28(\mathrm{~m}, 2 \mathrm{H}), 8.25-8.24(\mathrm{~m}, 1 \mathrm{H}), 8.20(\mathrm{~d}, J 7.4 \mathrm{~Hz}, 1 \mathrm{H}), 7.90(\mathrm{t}, J 7.0 \mathrm{~Hz}, 1 \mathrm{H}), 7.83-7.78(\mathrm{~m}$, $3 \mathrm{H}), 4.49$ (quartet, J $7.1 \mathrm{~Hz}, 2 \mathrm{H}), 1.43(\mathrm{t}, J 7.1 \mathrm{~Hz}, 3 \mathrm{H}) .{ }^{13} \mathrm{C} \mathrm{NMR}\left(100 \mathrm{MHz}, \mathrm{CDCl}_{3}\right): \delta_{\mathrm{c}} 162.7,159.5,133.3,132.0$, 130.7, 130.6, 130.3, 130.0, 129.9, 128.7, 128.6, 128.4, 128.0, 127.7, 127.6, 127.4, 127.1, 126.8, 125.6, 123.5, 121.6, 121.1, 103.0, 62.8, 14.3. ESI-MS $\mathrm{m} / \mathrm{z}$ calcd 401.1. Found: $402.1[\mathrm{M}+\mathrm{H}]^{+}, 419.1\left[\mathrm{M}+\mathrm{NH}_{4}\right]^{+}$. Anal. calcd for $\mathrm{C}_{28} \mathrm{H}_{19} \mathrm{NO}_{2}$ : C, 83.77; $\mathrm{H}, 4.77 ; \mathrm{N}, 3.49$. Found: $\mathrm{C}, 83.68 ; \mathrm{H}, 4.68 ; \mathrm{N}, 3.42$.

2-(Picen-13-ylmethylene) malononitrile (12). Prepared from 10 and malononitrile. Orange powder, Yield: 90\%. mp 258-260 ${ }^{\circ} \mathrm{C}$. IR (solid, $\mathrm{KBr}$, vmax, $\mathrm{cm}^{-1}$ ): 3437, 3048, 2226, 1561, 1382, 798. ${ }^{1} \mathrm{H}$ NMR (400 MHz, DMSO$\left.d_{6}\right): \delta_{H} 9.54(\mathrm{~s}, 1 \mathrm{H}), 9.34(\mathrm{~s}, 1 \mathrm{H}), 9.04-8.98(\mathrm{~m}, 3 \mathrm{H}), 8.42(\mathrm{~d}, J 7.6 \mathrm{~Hz}, 1 \mathrm{H}), 8.28-8.24(\mathrm{~m}, 2 \mathrm{H}), 8.22-8.17(\mathrm{~m}, 2 \mathrm{H})$, 7.87-7.79 (m, 4H). ${ }^{13} \mathrm{C}$ NMR (100 MHz, DMSO- $\left.d_{6}\right): \delta_{c} 165.4,132.9,131.7,130.4,130.2,129.9,129.6,129.3$, $129.1,128.8,128.6,128.2,128.0,127.7,127.5,127.4,126.9,126.6,126.0,123.5,121.9,121.7,114.4,113.8$, 84.9. ESI-MS $m / z$ calcd 354.1. Found: $353.0[\mathrm{M}-\mathrm{H}]^{-}$. Anal. calcd for $\mathrm{C}_{26} \mathrm{H}_{14} \mathrm{~N}_{2}: \mathrm{C}, 88.11 ; \mathrm{H}, 3.98 ; \mathrm{N}, 7.90$. Found: C, 88.10; H, 3.92; N, 7.88. 
2-Cyano-3-(picen-13-yl)acrylamide (13). Prepared from 10 and cyanoacetamide. Yellow powder, Yield: 82\%.

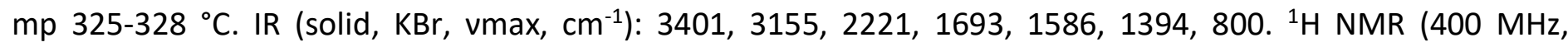
DMSO- $\left.d_{6}\right): \delta_{H} 9.43(\mathrm{~s}, 1 \mathrm{H}), 9.04-8.99(\mathrm{~m}, 4 \mathrm{H}), 8.43(\mathrm{~s}, 1 \mathrm{H}), 8.27-8.24(\mathrm{~m}, 3 \mathrm{H}), 8.18-8.12(\mathrm{~m}, 2 \mathrm{H}), 7.97(\mathrm{~s}, 1 \mathrm{H})$, $7.87(\mathrm{~m}, 1 \mathrm{H}), 7.80(\mathrm{~m}, 3 \mathrm{H}) .{ }^{13} \mathrm{C}$ NMR $(100 \mathrm{MHz}$, DMSO-d $): \delta_{c} 163.1,155.6,133.3,132.2,130.3,130.1,129.9$, 129.8, 129.6, 129.3, 129.2, 129.1, 128.3, 128.1, 127.9, 127.7, 127.5, 127.4, 125.6, 123.8, 122.6, 122.2, 117.3, 109.5. ESI-MS $m / z$ calcd 372.1. Found: $390.1\left[\mathrm{M}+\mathrm{NH}_{4}\right]^{+}$. Anal. calcd for $\mathrm{C}_{26} \mathrm{H}_{16} \mathrm{~N}_{2} \mathrm{O}: \mathrm{C}, 83.85 ; \mathrm{H}, 4.33 ; \mathrm{N}, 7.52$. Found: C, 83.78; $\mathrm{H}, 4.28 ; \mathrm{N}, 7.48$.

Diethyl 2-(picen-13-ylmethylene) malonate (14). Prepared from 10 and diethylmalonate. Pale yellow powder. Yield 75\%. $\mathrm{mp} 135-138{ }^{\circ} \mathrm{C}$. IR (solid, $\mathrm{KBr}$, vmax, $\mathrm{cm}^{-1}$ ): 3430, 3053, 2979, 1720, 1626, 1446, 1072, 807, 780. ${ }^{1 \mathrm{H}}$ NMR $\left(400 \mathrm{MHz}, \mathrm{CDCl}_{3}\right): \delta_{\mathrm{H}} 8.98(\mathrm{~s}, 1 \mathrm{H}), 8.81-8.68(\mathrm{~m}, 4 \mathrm{H}), 8.65(\mathrm{~s}, 1 \mathrm{H}), 8.10-8.00(\mathrm{~m}, 4 \mathrm{H}), 7.78-7.67(\mathrm{~m}, 4 \mathrm{H})$, 4.48 (quartet, J $7.1 \mathrm{~Hz}, 2 \mathrm{H}$ ), 4.26 (quartet, J $7.1 \mathrm{~Hz}, 2 \mathrm{H}$ ), 1.45 (t, J $7.1 \mathrm{~Hz}, 3 \mathrm{H}$ ), 1.04 (t, J 7.1 Hz, 3H). ${ }^{13} \mathrm{C} \mathrm{NMR}$ $\left(100 \mathrm{MHz}_{\mathrm{CDCl}}\right): \delta_{\mathrm{C}} 167.6,164.5,148.4,133.2,132.1,130.8,130.6,130.0,129.4,129.1,128.6,128.2,127.9$,

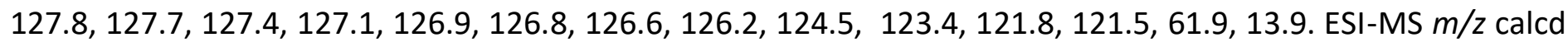
448.16. Found: $449.3[\mathrm{M}+\mathrm{H}]^{+}, 466.4\left[\mathrm{M}+\mathrm{NH}_{4}\right]^{+}$. Anal. calcd for $\mathrm{C}_{30} \mathrm{H}_{24} \mathrm{O}_{4}: \mathrm{C}, 80.34 ; \mathrm{H}, 5.39$. Found: $\mathrm{C}, 80.28 ; \mathrm{H}$, 5.28 .

Ethyl 3-oxo-2-(picen-13-ylmethylene)butanoate (15). Prepared from 10 and ethyl acetoacetate. Colourless powder, Yield: $75 \%$. mp 178-180 ${ }^{\circ} \mathrm{C}$. IR (solid, $\mathrm{KBr}, \mathrm{vmax}, \mathrm{cm}^{-1}$ ): 3435, 3052, 2981, 1727, 1663, 1424, 1377, 1237, 1049, 807, 778. ${ }^{1} \mathrm{H}$ NMR $\left(400 \mathrm{MHz}, \mathrm{DMSO}-d_{6}\right): \delta_{\mathrm{H}} 9.05-8.98(\mathrm{~m}, 2 \mathrm{H}), 8.90(\mathrm{~s}, 1 \mathrm{H}), 8.69-8.68(\mathrm{~m}, 3 \mathrm{H})$, 8.26-8.22 (m, 3H), $8.16(\mathrm{~m}, 1 \mathrm{H}), 7.86-7.83(\mathrm{~m}, 2 \mathrm{H}), 7.81-7.77(\mathrm{~m}, 2 \mathrm{H}), 4.12$ (quartet, $J .0 \mathrm{~Hz}, 2 \mathrm{H}), 2.51(\mathrm{~s}, 3 \mathrm{H})$, 0.91 (t, J 7.1 Hz, 3H). ${ }^{13} \mathrm{C}$ NMR (100 MHz, DMSO-d $\left.)_{6}\right): \delta_{c} 195.8,167.1,147.2,134.8,132.8,131.7,130.7,129.9$, 129.6, 129.5, 129.1, 128.8, 128.7, 128.6, 127.8, 127.5, 127.2, 127.1, 126.8, 123.4, 122.6, 122.1, 121.8, 61.1, 26.9, 13.6. ESI-MS $\mathrm{m} / \mathrm{z}$ calcd 418.15. Found: $419.3[\mathrm{M}+\mathrm{H}]^{+}$. Anal. calcd for $\mathrm{C}_{29} \mathrm{H}_{22} \mathrm{O}_{3}: \mathrm{C}, 83.23 ; \mathrm{H}, 5.30$. Found: $\mathrm{C}$, 83.38; $H, 5.26$.

3-(Picen-13-ylmethylene) pentane-2,4-dione (16). Prepared from 10 and acetylacetone. Brown powder, Yield: 70\%. mp 185-188 ${ }^{\circ} \mathrm{C}$. IR (solid, KBr, vmax, $\mathrm{cm}^{-1}$ ): 3435, 3051, 2920, 2850, 1706, 1655, 1377, 1236, 809, 778. ${ }^{1} \mathrm{H}$ NMR $\left(400 \mathrm{MHz}, \mathrm{DMSO}-d_{6}\right): \delta_{\mathrm{H}} 9.04(\mathrm{~d}, J 9.3 \mathrm{~Hz}, 1 \mathrm{H}), 8.98(\mathrm{~d}, J 9.2 \mathrm{~Hz}, 1 \mathrm{H}), 8.83(\mathrm{~d}, J 8.3 \mathrm{~Hz}, 1 \mathrm{H}), 8.77(\mathrm{~s}, 1 \mathrm{H})$, $8.71(\mathrm{~d}, J 8.2 \mathrm{~Hz}, 1 \mathrm{H}), 8.59(\mathrm{~s}, 1 \mathrm{H}), 8.27-8.20(\mathrm{~m}, 2 \mathrm{H}), 8.17-8.15(\mathrm{~m}, 2 \mathrm{H}), 7.87-7.81(\mathrm{~m}, 2 \mathrm{H}), 7.79-7.75(\mathrm{~m}, 2 \mathrm{H})$, $2.62(\mathrm{~s}, 3 \mathrm{H}), 2.32(\mathrm{~s}, 3 \mathrm{H}) .{ }^{13} \mathrm{C}$ NMR (100 MHz, DMSO- $\left.d_{6}\right): \delta_{c} 204.9,197.7,145.9,142.5,133.3,132.2,131.2$, 130.5, 130.1, 129.9, 129.5, 129.2, 129.1, 128.2, 128.0, 127.9, 127.6, 127.4, 127.2, 124.8, 123.2, 122.5, 122.2, 32.1 27.7. ESI-MS $m / z$ Calcd 388.1; found: $389.3[\mathrm{M}+\mathrm{H}]^{+}$. Anal. calcd for $\mathrm{C}_{28} \mathrm{H}_{20} \mathrm{O}_{2}$ : C, 86.57; H, 5.19. Found: C, 86.48; $\mathrm{H}, 5.25$.

5,6-Dimethoxy-2-(picen-13-ylmethylene)-2,3-dihydro-1H-inden-1-one (17). Prepared from 10 and 5,6 dimethoxy-1-indanone. Yellow powder, Yield: 85\%. m.p. 292-294 ${ }^{\circ} \mathrm{C}$. IR (solid, $\mathrm{KBr}$, vmax, $\mathrm{cm}^{-1}$ ): 3435,3051 , 1684, 1499, 1304, 1129, 1096, 804. ${ }^{1} \mathrm{H}$ NMR $\left(400 \mathrm{MHz}, \mathrm{CDCl}_{3}+\mathrm{DMSO}_{-} d_{6}\right): \delta_{\mathrm{H}} 9.05(\mathrm{~s}, 1 \mathrm{H}), 8.82(\mathrm{~d}, \mathrm{~J} 9.2 \mathrm{~Hz}, 2 \mathrm{H})$, 8.77-8.74 (m, 2H) $8.46(\mathrm{~s}, 1 \mathrm{H}), 8.09-8.06(\mathrm{~m}, 2 \mathrm{H}), 8.04-8.02(\mathrm{~m}, 2 \mathrm{H}), 7.79-7.75(\mathrm{~m}, 1 \mathrm{H}), 7.72-7.69(\mathrm{~m}, 1 \mathrm{H}), 7.68-$ $7.65(\mathrm{~m}, 1 \mathrm{H}), 7.64-7.58(\mathrm{~m}, 1 \mathrm{H}), 7.45(\mathrm{~s}, 1 \mathrm{H}), 7.05(\mathrm{~s}, 1 \mathrm{H}), 4.14(\mathrm{~s}, 2 \mathrm{H}), 4.00(\mathrm{~s}, 6 \mathrm{H}) .{ }^{13} \mathrm{C} \mathrm{NMR}\left(100 \mathrm{MHz}_{\mathrm{CDCl}}+\right.$ DMSO- $\left.d_{6}\right): \delta_{C} 192.7,155.5,149.7,145.6,136.9,135.3,132.9,132.1,131.9,131.5,130.6,130.2,129.9,128.7$, 128.5, 128.4, 128.3, 128.2, 128.1, 127.6, 127.2, 127.0, 126.6, 126.3, 124.1, 122.9, 121.6, 121.5, 107.6, 105.1, 56.3, 56.1, 31.32, 29.6. ESI-MS $\mathrm{m} / \mathrm{z}$ calcd 480.17. Found: $481.4[\mathrm{M}+\mathrm{H}]^{+}$. Anal. calcd for $\mathrm{C}_{34} \mathrm{H}_{24} \mathrm{O}_{3}: \mathrm{C}, 84.98 ; \mathrm{H}$, 5.03. Found: C, 84.88; H, 5.18 .

In vitro anti-inflammatory activity. The in vitro anti-inflammatory activity of synthesized compounds was studied using bovine serum albumin denaturation method. ${ }^{29,30}$ In brief, increasing concentrations of the test or reference compound were incubated with $0.5 \% \mathrm{w} / \mathrm{v}$ of bovine serum albumin at $37{ }^{\circ} \mathrm{C}$ for 20 minutes and 
the temperature was increased to keep the samples at $57{ }^{\circ} \mathrm{C}$ for 30 minutes. After cooling to room temperature, the turbidity was measured using UV-Visible spectrophotometer at $660 \mathrm{~nm}$ following addition of phosphate buffered saline. The control represents $100 \%$ protein denaturation. The results were compared with reference drug Diclofenac sodium. The percentage inhibition of protein denaturation by the drug was calculated by using the following formula.

Percentage Inhibition $=100$-[(optical density of test solution-optical density of product control) $\div$ (optical density of test control)] $\times 100$

\section{Acknowledgements}

The authors are thankful to the management of Orchid Pharma Limited, Chennai- 600 119, India and Rama Krishna Mission Vivekananda College, Chennai-600 004, India for providing facilities.

\section{References}

1. Mitsuhashi, R.; Suzuki, Y.; Yamanari, Y.; Mitamura, H.; Kambe, T.; Ikeda, N.; Okamoto H.; Fujiwara, A.; Yamaji, M.; Kawasaki, N.; Maniwa, Y.; Kubozono, Y. Nature 2010, 464, 76. https://doi.org/10.1038/nature08859

2. Okamoto, H.; Kawasaki, N.; Kaji, Y.; Kubozono, Y.; Fujiwara, A.; Yamaji, M. J. Am. Chem. Soc. 2008, 130 10470. https://doi.org/10.1021/ja803291a

3. Pentacyclic Triterpenes as Promising Agents in Cancer Salvador, J. A. R., Ed., Nova Science: New York, 2010.

4. De Silva, M. L.; David, J. P.; Silva, L. C.; Santos, R. A.; David, J. M.; Lima, L. S.; Reis, P. S.; Fontana, R. Molecules 2012, 17, 12197.

https://doi.org/10.3390/molecules171012197

5. Lin, K. W.; Huang, A. M.; Lin, C. C.; Chang, C. C.; Hsu, W. C.; Hour, T. C.; Pu, Y. S.; Lin, C. N. Eur. J. Pharmacol. 2014, 740, 742.

https://doi.org/10.1016/j.ejphar.2014.05.051

6. Galaĭko, N. V.; Tolmacheva, I. A.; Grishko, V. V.; Volkova, L. V.; Prevozchikova, E. N.; Pestereva, S. A. Bioorganicheskaya Khimiya 2010, 36, 556.

7. Putta, S.; Yarla, N. S.; Kilari, E. K.; Surekha, C.; Aliev, G.; Divakara, M. B.; Santosh, M. S.; Ramu, R.; Zameer, F.; Mn, N. P.; Chintala, R.; Rao, P. V.; Shiralgi, Y.; Dhananjaya, B. L. Curr. Top. Med. Chem. 2016, 16, 2532.

https://doi.org/10.2174/1568026616666160414123343

8. Safayhi, H.; Sailer, E. R. Planta Med. 1997, 63, 487.

https://doi.org/10.1055/s-2006-957748

9. Ray, S. D.; Ray, S.; Zia-Ul-Haq, M.; De Feo, V.; Dewanjee, S. BMC Complementary and Alternative Medicine 2015, 15, 416.

https://doi.org/10.1186/s12906-015-0942-7

10. Sarbani Dey Ray.; Saikat Dewanjee. Natural Product Research 2014.

https://doi.org/10.1080/14786419.2014.983921 
11. Shashank Kumar; Abhay Pandey K. The Scientific World Journal, 2013.

https://doi.org/10.1155/2013/162750

12. El-Gazzar, A. B.; Hafez, H. N.; Abu-Hashem A. A.; Aly, A. S. Phosphorus, Sulfur, and Silicon and the Related Elements 2009, 184, 379.

https://doi.org/10.1080/10426500802167027

13. Badger, G. M. J. Chem. Soc. 1941, 535.

https://doi.org/10.1039/jr9410000535

14. Everett, J. L.; Kon, G. A. R. J. Chem. Soc. 1948, 1601.

https://doi.org/10.1039/jr9480001601

15. Herbert, R. B.; Moody, C. J. J. Chem. Soc. D, Chem. Comm. 1970, 2, 121.

https://doi.org/10.1039/C29700000121

16. Iwao, M.; Lee, M. L.; Castle, R. N. J. Het. Chem. 1980, 17, 1259.

https://doi.org/10.1002/jhet.5570170623

17. Mallory, F. B.; Mallory, C. W. Photocyclization of Stilbenes and Related Molecules. In Organic Reactions, John Wiley: New York, 2004; Vol. 30, p 1.

18. Yang, S-M.; Shie, J-J.; Fang, J-M.; Nandy, S. K.; Chang, H-Y.; Lu, S-H.; Wang, G. J. Org. Chem. 2002, 67, 5208.

https://doi.org/10.1021/jo0257849

19. Baldwin, L. J.; Tedjamulia, M. L.; Stuart, J. G.; Castle, R. N.; Lee, M. L. J. Het. Chem. 1984, $21,1775$. https://doi.org/10.1002/jhet.5570210642

20. Dopper, J. H.; Oudman, D.; Wynberg, H. J. Org. Chem. 1975, 40, 3398.

https://doi.org/10.1021/jo00911a020

21. Vingiello, F. A.; Yanez, J.; Campbell, J. A. J. Org. Chem. 1971, 36, 2053.

https://doi.org/10.1021/j000814a005

22. Liepa, A. J.; Summons, R. E. J. Chem. Soc., Chem. Comm. 1977, 22, 826. https://doi.org/10.1039/C39770000826

23. Elango, S.; Venugopal, M.; Suresh, P. S.; Eni. Tetrahedron 2005, 61, 1443.

https://doi.org/10.1016/j.tet.2004.12.005

24. Dineen, T. A.; Roush, W. R. Org. Lett. 2004, 6, 2043.

https://doi.org/10.1021/ol049331x

25. Brown, H. C.; Rao, C. G.; Kulkarni, S. U. J. Org. Chem. 1979, 44, 2809.

https://doi.org/10.1021/jo01329a051

26. Corey, E. J.; Suggs, J. W. Tetrahedron Lett. 1975, 16, 2647.

https://doi.org/10.1016/S0040-4039(00)75204-X

27. Meguro, K.; Fujita, T.; Hatanaka, C.; Ooi, S. U.S. Patent 4812 570, 1989.

28. Sohda, T.; Mizuno, K.; Momose, Y.; Ikeda, H.; Fujita, T. Meguro, K. J. Med. Chem. 1992, 35, 2617. https://doi.org/10.1021/jm00092a012

29. Mizushima, Y.; Kobayashi, M. J. Pharm. Pharmacol. 1968, 20, 169.

https://doi.org/10.1111/j.2042-7158.1968.tb09718.x

30. Rajadurai, R.; Padmanabhan, R.; Ananthan, S. Med. Chem. Res. 2013, 22, 4164. https://doi.org/10.1007/s00044-012-0410-6 